\title{
Prevalência de Staphylococcus spp resistentes à meticilina isolados em uma maternidade escola da Cidade de Natal, Estado do Rio Grande do Norte
}

\author{
Prevalence of methicillin-resistant Staphylococcus spp isolated in a teaching \\ maternity hospital in the City of Natal, State of Rio Grande do Norte
}

\author{
Francisco Canindé de Sousa Junior ${ }^{1}$, Éderson William de Figueiredo Nunes ${ }^{1}$, \\ Ermeton Duarte do Nascimento ${ }^{1}$, Solange Maria de Oliveira ${ }^{2}$, \\ Maria Celeste Nunes de Melo ${ }^{1}$ e Maria José de Britto Costa Fernandes ${ }^{1}$
}

\begin{abstract}
RESUMO
Com o objetivo de determinar a prevalência de Staphylococcus spp resistentes à meticilina isolados na Maternidade Escola Januário Cicco, Natal, RN, no período de 2002 a 2003, analisou-se 1.576 materiais clínicos de pacientes hospitalizados. As amostras foram coletadas, processadas e identificadas conforme procedimento padrão para cada espécime clínico. 0 perfil de susceptibilidade in vitro foi realizado pelo método de Kirby-Bauer. Isolou-se 188 cepas de Staphylococcus spp, das quais 105 foram identificadas como Staphylococcus aureus e 83 como Staphylococcus coagulase negativos. Staphylococcus aureus foi isolado com mais frequiência em secreções enquanto Staphylococcus coagulase negativos foram mais prevalentes em hemoculturas. A elevada (41,5\%) prevalência dos Staphylococcus spp resistentes à meticilina demonstra a necessidade de medidas profiláticas imediatas com o objetivo de impedir a disseminação desse fenômeno.
\end{abstract}

Palavra-chaves: Staphylococcus. Prevalência. Resistência à meticilina. Testes de sensibilidade microbiana.

\begin{abstract}
Analyses on 1,576 clinical specimens from hospitalized patients were performed with the aim of determining the prevalence of methicillin-resistant Staphylococcus spp isolated in the Januário Cicco Teaching Maternity Hospital in Natal, Rio Grande do Norte, in 2002 and 2003. The samples were collected, processed and identified according to standard procedures, for each clinical specimen. In vitro susceptibility profiles were obtained using the Kirby-Bauer method. 188 strains of Staphylococcus spp were isolated: 105 were identified as Staphylococcus aureus and 83 as coagulase-negative Staphylococcus. Staphylococcus aureus was isolated most often in secretions, whereas coagulase-negative Staphylococcus was most prevalent in blood cultures. The high (41.5\%) prevalence of methicillin-resistant Staphylococcus spp shows the need for immediate prophylactic measures aimed at impeding the dissemination of this phenomenon.
\end{abstract}

Key-words: Staphylococcus. Prevalence. Methicillin resistance. Microbial sensitivity tests.

A primeira cepa de Staphylococcus spp resistente à meticilina foi detectada na Europa em 1961 e pertencia a espécie Staphylococcus aureus ${ }^{18}$. Nas décadas seguintes os Staphylococcus aureus resistentes à meticilina (MRSA) se tornaram um grave problema de saúde pública mundial ${ }^{20}$. Atualmente, os MRSA são importantes agentes de infecções hospitalares e comunitárias ${ }^{8}$. No Brasil, 40 a $60 \%$ dos Staphylococcus aureus isolados de amostras provenientes de pacientes hospitalizados são resistentes à meticilina ${ }^{7}$. Nestes

1. Departamento de Microbiologia e Parasitologia, Universidade Federal do Rio Grande de Norte, Natal, RN. 2. Maternidade Escola Januário Cicco, Universidade Federal do Rio Grande de Norte, Natal, RN.

Endereço para correspondência: Dra. Maria José de Britto Costa Fernandes. Dept $^{\circ}$ de Microbiologia e Parasitologia/Centro de Biociências/UFRN. Campus Universitário s/n, 59072-970 Natal, RN.

Tel: $55843215-3433$

e-mail: majofernandes@gmail.com

Recebido para publicação em 17/12/2007

Aceito em 13/02/2009 microrganismos, a resistência é resultado do gene cromossômico $m e c A$, que produz uma nova proteína ligadora de penicilina (PBP) com baixa afinidade pelos beta-lactâmicos ${ }^{18}$.

Além dos MRSA, outras cepas do gênero têm sido isoladas de infecções hospitalares ${ }^{3}$. 0 isolamento de Staphylococcus coagulase negativa (CNS), tem sido descrito e demonstra que esse grupo tem desenvolvido resistência à vários antimicrobianos, inclusive à meticilina ${ }^{14}{ }^{16}$. Dentre as espécies de CNS, Staphylococcus epidermidis é a mais prevalente em bacteremias, situando-se entre 74\% e 92\% dos Staphylococcus spp isolados em hemoculturas ${ }^{4}$.

Com o crescimento da caracterização de infecção por CNS, o interesse em estudar sua susceptibilidade aos antimicrobianos também tem aumentado ${ }^{2}$. A elevada transmissibilidade de genes de resistência entre linhagens e o uso abusivo de drogas antimicrobianas têm se constituído em importantes fatores na seleção de amostras multirresistentes ${ }^{2}$. Associados ao mecanismo de resistência à meticilina também podem ser citados a produção de invasinas, toxinas e a formação de biofilme . $^{8}$ 
Atualmente, no Brasil, a situação da resistência dos Staphylococcus à meticilina, e conseqüentemente à oxacilina, já que ambos possuem o mesmo mecanismo de ação, têm apresentado uma grande variação entre os estados de uma mesma região e entre os hospitais analisados no mesmo estado, devendo ser avaliada localmente ${ }^{16}$.

Vários trabalhos realizados em maternidades-escola avaliam os riscos que envolvem a mãe e a criança quando da necessidade de procedimentos invasivos, do tipo de parto adotado pela equipe médica ou até mesmo da permanência dos pacientes no ambiente hospitalar, fazendo a correlação com processos infecciosos antes, durante e depois do parto ${ }^{117}$. Segundo Tsu e cols ${ }^{17}$, a sepse está entre as principais causas de morte materna nesses hospitais. Em Unidades Pediátricas de Terapia Intensiva o maior problema enfrentado pelas equipes está no risco potencial de bacteremia entre os neonatos de baixo peso $(<1.500 \mathrm{~g})$, que são imunologicamente imaturos e freqüentemente também necessitam de procedimentos invasivos para administração de substâncias nutritivas e medicamentosas ${ }^{2}$.

Este trabalho tem como objetivo avaliar a prevalência de Staphylococcus spp resistentes à meticilina isolados na Maternidade Escola Januário Cicco, Natal, RN, no período de 2002 a 2003.

\section{MATERIAL E MÉTODOS}

Foram avaliados 1.576 materiais clínicos de pacientes hospitalizados na Maternidade Escola Januário Cicco (MEJC) da Universidade Federal do Rio Grande do Norte (UFRN), Natal, RN, no período de janeiro de 2002 a dezembro de 2003. As amostras foram coletadas de pacientes com suspeita clínica de infecção, conforme procedimento padrão para cada espécime clínico. Utilizaram-se frascos de hemocultura adulto e pediátrico contendo BHI para coleta de sangue, frascos estéreis para coleta de ponta de cateter e swabs e meio Stuart para coleta de secreções de ferida operatória, ocular e de ouvido.

Todas as hemoculturas culturas foram submetidas à subcultura em ágar sangue de carneiro a $5 \%$ e ágar chocolate, a $35^{\circ} \mathrm{C}$, após $24 \mathrm{~h}$, $48 \mathrm{~h}$ e 7 dias de incubação no BHI. As secreções foram semeadas em ágar sangue de carneiro a 5\% e ágar manitol salgado. Para as pontas de cateter, foi utilizada a técnica Semiquantitativa de Maki e cols ${ }^{6}$ em ágar sangue de carneiro 5\%, em que foram consideradas positivas culturas com contagem igual ou superior a 15 unidades formadoras de colônia (UFC).

Para identificação, utilizou-se a técnica de coloração de Gram, fermentação do manitol, e testes para avaliar a produção das enzimas catalase e coagulase. 0 perfil de susceptibilidade a antimicrobianos in vitro foi realizado pelo método de KirbyBauer, seguindo protocolos Clinical and Laboratory Standards Institute (CLSI). Para avaliar o perfil de resistência à meticilina, observou-se a sensibilidade da cepa frente ao disco de oxacilina $(1 \mu \mathrm{g})$, em que cepas com halo de inibição menor ou igual a $10 \mathrm{~mm}$ para oxacilina foram consideradas resistentes à meticilina. Os dados foram descritos em frequiências absolutas e percentuais, e para avaliar a diferença entre as frequiências observadas utilizouse o teste estatístico do qui-quadrado utilizando-se o software Epi Info 6.2.

\section{RESULTADOS}

De uma casuística de 1.576 amostras clínicas, 188 apresentaram crescimento de Staphylococcus spp. Destas, 105 foram identificadas como Staphylococcus aureus e 83 como Staphylococcus coagulase negativa (CNS). Na Tabela 1, está descrita a distribuição das cepas de Staphylococcus aureus e CNS isoladas de hemoculturas e secreção, de acordo com o ano estudado.

Ao se correlacionar o isolamento de Staphylococcus spp com o tipo de amostra, observou-se que Staphylococcus aureus foi mais frequiente em secreções enquanto CNS foi mais prevalente em hemoculturas $(\mathrm{P}<0,001)$.

A avaliação da susceptibilidade das cepas à meticilina mostrou uma prevalência de resistência de 18,1\% para Staphylococcus aureus e $71,1 \%$ para o grupo dos CNS. A análise de todos os isolados de Staphylococcus spp mostrou uma prevalência 41,5\% para cepas resistentes à meticilina, como mostra a Tabela 2.

\section{TABELA 1}

Frequiência de Staphylococcus aureus e Staphylococcus coagulase negativa isolados de hemoculturas e secreções na Maternidade Escola Januário Cicco.

\begin{tabular}{|c|c|c|c|c|c|c|c|c|}
\hline \multirow[b]{3}{*}{ Ano } & \multicolumn{4}{|c|}{ Hemocultura } & \multicolumn{4}{|c|}{ Secreção } \\
\hline & \multicolumn{2}{|c|}{ S. aureus } & \multicolumn{2}{|c|}{ CNS } & \multicolumn{2}{|c|}{ S. aureus } & \multicolumn{2}{|c|}{ CNS } \\
\hline & $\overline{\mathrm{n}^{0}}$ & $\%$ & $\mathrm{n}^{0}$ & $\%$ & $\overline{\mathrm{n}^{0}}$ & $\%$ & $\overline{\mathrm{n}^{0}}$ & $\%$ \\
\hline 2002 & 5 & 50,0 & 14 & 38,9 & 40 & 42,1 & 24 & 51,1 \\
\hline 2003 & 5 & 50,0 & 22 & 61,1 & 55 & 57,9 & 23 & 48,9 \\
\hline Total & 10 & 100,0 & 36 & 100,0 & 95 & 100,0 & 47 & 100,0 \\
\hline
\end{tabular}

S. aureus: Staphylococcus aureus, CNS: Staphylococcus coagulase negativa, $\leq 0,05$ (qui quadrado)

TABELA 2

Prevalência de Staphylococcus aureus e CNS resistentes à meticilina isolados na Maternidade Escola Januário Cicco

\begin{tabular}{lcccc}
\hline Isolado & $\begin{array}{c}\text { Total } \\
\text { de cepas }\left(\mathrm{n}^{\mathrm{o}}\right)\end{array}$ & $\begin{array}{c}\text { Cepas resistentes } \\
\text { à meticilina }\end{array}$ & Prevalência & $\begin{array}{c}\text { Intervalo } \\
\text { de confiança } 95 \%\end{array}$ \\
\hline S. aureus & 105 & 19 & 18,1 & 14,3 a 21,9 \\
CNS & 83 & 59 & 71,1 & 66,1 a 76,1 \\
\hline Total & $\mathbf{1 8 8}$ & $\mathbf{7 8}$ & $\mathbf{4 1 , 5}$ & $\mathbf{3 7 , 9}$ a 45,1 \\
\hline
\end{tabular}

S. aureus: Staphylococcus aureus, CNS: Staphylococcus coagulase negativa.

\section{DISCUSSÃO}

O presente estudo fundamentou-se na avaliação da prevalência de Staphylococcus spp resistentes à meticilina isolados em uma maternidade escola da Cidade do Natal, RN. Nesse estudo os agentes mais frequientes nas hemoculturas foram os Staphylococcus coagulase negativa, o que pode ser consequiência do uso de dispositivos intravenosos, como cateteres. Os cateteres vasculares são importantes fontes de infecção, por serem 
facilmente colonizados por microrganismos da pele presentes ao longo do local de inserção, especialmente CNS, microrganismos prevalentes como microbiota da pele e mucosas ${ }^{2}$.

Os nossos resultados apresentaram, para as secreções, uma pequena frequiência de CNS quando comparado a incidência de Staphylococcus aureus. No Brasil, Staphylococcus aureus é o microrganismo mais freqüentemente isolado em infecções hospitalares ${ }^{11}$, sendo também o maior responsável pela mastite puerperal em maternidades ${ }^{15}$. Este patógeno tem ainda se destacado em infecções comunitárias, sendo a principal causa daquelas adquiridas na comunidade em vários países ${ }^{20}$.

Staphylococcus coagulase negativa apresentam elevado risco potencial de bacteremia nosocomial entre recém-nascidos de baixo peso em UTIs pediátricas. 0 aumento da incidência desses processos infecciosos, nos últimos 20 anos, tem sido também associado a maior sobrevida de crianças prematuras de baixo peso ao nascimento e a sua longa permanência no ambiente hospitalar².

Em uma pesquisa realizada num Hospital Escola de Uberaba, foram isoladas cepas de Staphylococcus aureus em $87 \%$ dos colchões dos leitos da UTI, demonstrando a necessidade de um maior controle do processo de limpeza e desinfecção dos mesmos ${ }^{9}$.

Vários trabalhos relatam o envolvimento de CNS em processos infecciosos de próteses, implantes, cateteres venosos e outros dispositivos cirúrgicos, porém o isolamento de cepas de Staphylococcus epidermidis em hemoculturas tem se tornado rotina na maioria dos hospitais, e revela ser esse um dos principais agentes causadores de bacteremia ${ }^{8}$.

Em nosso trabalho, quando comparamos o número de casos de microrganismos resistentes à meticilina, verificamos que os CNS apresentam uma maior prevalência de cepas resistentes que Staphylococcus aureus $(71,1 \%$ frente a $18,1 \%)$. 0 programa SENTRY encontrou uma proporção de resistência à meticilina em torno de 34\% para Staphylococcus aureus e $80 \%$ para CNS, em hospitais brasileiros no período de três anos ${ }^{13}$, mostrando que a resistência é mais pronunciada em CNS, embora não se possa desconsiderar a prevalência de Staphylococcus aureus.

O fenômeno da resistência tem sido atribuído ao uso indiscriminado de antimicrobianos, o que onera excessivamente o hospital, pelo aumento do uso de fármacos e da permanência dos pacientes infectados no hospital, aumentando ainda, o risco de contrair uma outra infecção nesse ambiente que possa levar esse paciente ao óbito5.

Segundo a Organização Mundial da Saúde (OMS), a mortalidade nas maternidades se correlaciona negativamente com o funcionamento do sistema de saúde, entretanto, no Brasil a notificação da mortalidade nesses hospitais ainda é precária, exigindo um maior empenho das autoridades ${ }^{18}$. Em estudo retrospectivo, realizado entre 1927 a 2001, numa Maternidade Escola de Juiz de Fora, MG, a infecção puerperal representou $15,4 \%$ das causas de morte materna nesse local ${ }^{1}$. Nesses ambientes, os processos infecciosos são mais comuns em pacientes submetidas a cesarianas do que aquelas submetidas ao parto transpélvico ${ }^{1012}$, o que indica que o processo cirúrgico é um dos fatores de risco nesses hospitais.
Os casos de mortalidade materna por infecção tiveram forte redução nos últimos 50 anos, mas ainda representam um dado preocupante, principalmente quando o agente etiológico é um organismo resistente ou multiresistente. A literatura internacional e as evidências em nosso meio, vêm mostrando a acentuada evolução do Staphylococcus aureus e do Staphylococcus coagulase negativa e a sua resistência à meticilina no ambiente hospitalar ${ }^{14}$. Vale ressaltar que sua importância não diz respeito apenas à freqüência, mas também ao seu potencial de gravidade nas infecções hospitalares e a sua forma de disseminaçã $0^{15}$. Esses aspectos têm fundamento e despertam preocupação, a passo que, assim como o paciente, a equipe que o acompanha pode estar colonizada pela cepa, especialmente na mucosa e na pele, o que acarreta uma maior chance de contaminação ou reinfecção ${ }^{8}$.

A frequiência e a evolução das infecções por Staphylococcus spp resistentes em indivíduos hospitalizados, especialmente em UTIs pediátricas e leitos de maternidades como um todo, demonstra a necessidade de medidas profiláticas imediatas com o objetivo de impedir a disseminação desse fenômeno, tais como: lavagem das mãos, racionalização do uso de antimicrobianos, desenvolvimento de um banco de dados nacional confiável, criação de laboratórios de referência para verificação da resistência e melhoria técnica dos laboratórios de microbiologia.

\section{REFERÊNCIAS}

1. Andrade ATL, Guerra MO, Andrade GN, Araújo DAC, Souza JP. Mortalidade materna: 75 anos de observações em uma Maternidade Escola. Revista Brasileira de Ginecologia e Obstetrícia 28:380-387, 2006.

2. Cunha MLRS, Lopes CAM. Estudo da produção de $\beta$-lactamase e sensibilidade às drogas em linhagens de estafilococos coagulase-negativos isolados de recémnascidos. Jornal Brasileiro de Patologia e Medicina Laboratorial 38:281-290, 2002.

3. Cunha MLRS, Lopes CAM, Rugolo LMSS, Chalita LVAS. Significância clínica de estafilococos coagulase-negativa isolados de recém-nascidos. Jornal de Pediatria 78:279-288, 2002.

4. Hudome SM, Fisher MC. Nosocomial infections in the neonatal intensive care. Current Opnion in Infectious Diseases 14: 303-307, 2001.

5. Kloosi WE, Bannerman TL. Update on Clinical Significance of Coagulase-Negative Staphylococci. Clinical Microbiology Reviews 7:117-140, 1994.

6. Maki DG, Weise CE, Sarafin HW. A semiquantitative culture method for identifying intravenous-catheter-related infection. The New England Journal of Medicine 296:1305-1309, 1977.

7. Melo MCN, Carvalho MCS, Ferreira RL, Coelho LR, Souza R, Gobbi CN, Rozenbaum R, Solari CA, Ferreira-Carvalho BT, Figueiredo AMS. Detection and molecular characterization of a gentamicin-susceptible, methicillin-resistant Staphylococcus aureus (MRSA) clone in Rio de Janeiro that resembles the New York/Japonese clone. Journal of Hospital Infection 58: 276-285, 2004.

8. Michelim L, Lahude M, Araújo PR, Giovanaz DSH, Müller G, Delamare APL, Costa SOP, Echeverrigaray S. Pathogenicity factors and antimicrobial resistance of Staphylococcus epidermidis associated with nosocomial infections occurring in intensive Care units. Brazilian Journal of Microbiology 36:17-23, 2005.

9. Mundim GJ, Dezena RA, Oliveira ACS, Silva PR, Cardoso M, Pereira GA, Morais CA, Terra APS. Avaliação da presença de Staphylococcus aureus nos leitos do Centro de Terapia Intensiva do Hospital Escola da Faculdade de Medicina do Triângulo Mineiro, em relação à posição no colchão antes e após a limpeza. Revista da Sociedade Brasileira de Medicina Tropical 36:685-688, 2003.

10. Nomura RMY, Alves EA, Zugaib M. Complicações maternas associadas ao tipo de parto em hospital universitário. Revista de Saúde Pública 38:9-15, 2004. 
11. Oliveira GA, Faria JB, LewCE, Mamizuka EM. Characterization of the Brazilian Endemic Clone of Methicillin-Resistant Staphylococcus aureus (MRSA) from Hospitals Throughout Brazil. The Brazilian Journal of Infectious Diseases 5:163-170, 2001.

12. Ramos JGL, Martins-Costa S, Vettorazzi-Stuczynski J, Brietzke E. Morte Materna em Hospital Terciário do Rio Grande do Sul - Brasil: um Estudo de 20 Anos. Revista Brasileira de Ginecologia e Obstetrícia 25:431-436, 2003.

13. Sader HS, Gales AC, Pfaller MA, Mendes RE, Zoccoli C, Barth A, Jones RN. Pathogen frequency and resistance patterns in brazilian hospitals: summary of results from three years of the SENTRY antimicrobial surveillance program. The Brazilian Journal of Infectious Diseases 5:200-214, 2001.

14. Sader HS, Streit JM, Fritsche TR, Jones RN. Antimicrobial susceptibility of Gram-positive bacteria isolated from European medical centres: results of the Daptomycin Surveillance Programme (2002-2004). Clinical Microbiology and Infection, 12:844-852, 2006

15. Sales AN, Vieira GO, Moura MSQ, Almeida SPTMA, Vieira T. Mastite Puerperal: Estudo de Fatores Predisponentes. Revista Brasileira de Ginecologia e Obstetrícia 22: 627-632, 2000
16. Tavares W. Bactérias gram-positivas problemas: resistência do estafilococo, do enterococo e do pneumococo aos antimicrobianos. Revista da Sociedade Brasileira de Medicina Tropical 33:281-301, 2000.

17. Tsu VD, Shane B. New and underutilized technologies to reduce maternal mortality: call to action from a Bellagio workshop. International Journal of Gynaecology and Obstetrics 85:S83-93, 2004

18. Vivone AM, Moreira BM. Application of molecular techniques in the study of Staphylococcus aureus clonal evolution - A Review. Memórias do Instituto Oswaldo Cruz 100: 693-698, 2005.

19. World Health Organization. Maternal mortality in 2000: estimates developed by WHO, UNICEF and UNFPA. Geneva: World Health Organization, 2004.

20. Zetola N, Francis JS, Nuermberger EI, Bishai WR. Community-acquired meticillinresistant Staphylococcus aureus: na emerging threat. Lancet Infection Diseases 5:275-286, 2005 